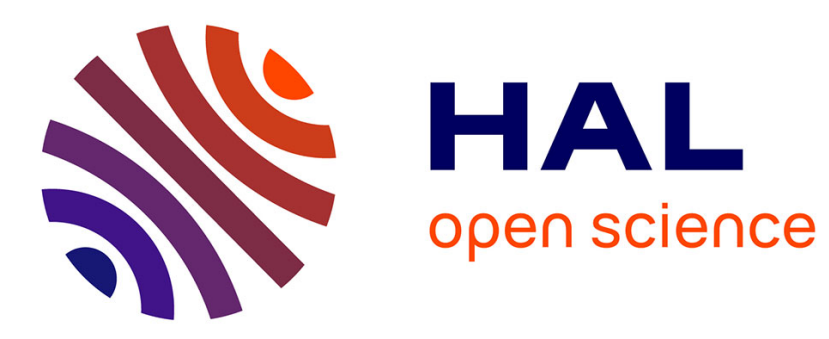

\title{
Hydrométrie et normalisation
}

Arnaud Belleville, Gilles Pierrefeu, Jérôme Le Coz, Frédérique Larrarte, Pierre Marchand, Mélanie Pinatton, Benedicte Augeard, Pierre-Marie Bechon, David Besson, Pascal Chisne, et al.

\section{- To cite this version:}

Arnaud Belleville, Gilles Pierrefeu, Jérôme Le Coz, Frédérique Larrarte, Pierre Marchand, et al.. Hydrométrie et normalisation. La Houille Blanche - Revue internationale de l'eau, 2017, 2017 (6), pp.57-66. 10.1051/lhb/2017059 . hal-01686544

\section{HAL Id: hal-01686544 https://hal.science/hal-01686544}

Submitted on 16 May 2020

HAL is a multi-disciplinary open access archive for the deposit and dissemination of scientific research documents, whether they are published or not. The documents may come from teaching and research institutions in France or abroad, or from public or private research centers.
L'archive ouverte pluridisciplinaire HAL, est destinée au dépôt et à la diffusion de documents scientifiques de niveau recherche, publiés ou non, émanant des établissements d'enseignement et de recherche français ou étrangers, des laboratoires publics ou privés. 


\title{
Hydrométrie et normalisation
}

\author{
Arnaud BELLEVILLE ${ }^{1}$, Gilles PIERREFEU², Jérôme LE $\mathrm{COZ}^{3}$, Frédérique LARRARTE4, \\ Pierre MARCHAND ${ }^{5}$, Mélanie PINATTON ${ }^{6}$, Bénédicte AUGEARD $^{7}$, Pierre-Marie BECHON ${ }^{8}$, \\ David BESSON ${ }^{9}$, Pascal CHISNE ${ }^{10}$, Guillaume DRAMAIS ${ }^{3}$, Christophe JOSSERAND ${ }^{11}$, \\ Stéphanie POLIGOT-PITSCH ${ }^{12}$, Rachel PUECHBERTY ${ }^{13}$
}

\author{
${ }^{I}$ EDF-DTG, 21 Avenue de l'Europe, BP 41, 38040 Grenoble, France, arnaud.belleville@edf.fr \\ ${ }^{2}$ CNR, 4 Rue de Chalon sur Saône,69007Lyon, France, g.pierrefeu@cnr.tm.f \\ ${ }^{3}$ IRSTEA, 5 Rue de la Doua, BP 32108, 69616 Villeurbanne Cedex, France, jerome.lecoz@irstea.fr \\ ${ }^{4}$ IFSTTAR, LUNAM, GERS-LEE, CS 4, 44344 Bouguenais Cedex, France, frederique.larrarte@ifsttar.fr \\ ${ }_{5}^{5}$ IRD, 911 Avenue Agropolis, BP 64501, 34394 Montpellier Cedex, France, pierre.marchand@umontpellier.fr \\ ${ }^{6}$ AFNOR Normalisation, 11 Rue Francis de Pressensé 93571 La Plaine Saint-Denis Cedex, France \\ ${ }^{7}$ ONEMA, Le Nadar Hall C, 5 Allée Félix Nadar, 94300 Vincennes, France \\ ${ }^{8}$ DREAL Auvergne Rhône Alpes, 5 Place Jules Ferry, 69006 Lyon, France \\ ${ }^{9}$ DREAL Centre Val de Loire, 5, Avenue Buffon, CS96407, 45064 Orléans Cedex 2, France \\ ${ }^{10}$ CACG, BP 449, Chemin de la Lalette, 65000 Tarbes Cedex, France \\ ${ }^{11}$ INERIS, Parc Technologique ALATA, BP2, Rue Jacques Taffanel, 60550 Verneuil-en-Halatte, France \\ 12 DREAL Pays de Loire, 5 Rue Françoise Giroud, 44200 Nantes, France \\ ${ }^{13}$ SCHAPI, 42 Avenue Gaspard Coriolis, 31057 Toulouse Cedex 01, France
}

RÉSUMÉ. - La normalisation permet de définir un langage commun entre les acteurs d'un domaine particulier, de clarifier, d'harmoniser les pratiques et de définir le niveau de qualité, de sécurité, de moindre impact environnemental des pratiques.

En France, dans le domaine de l'hydrométrie, les travaux de normalisation sont menés par la commission AFNOR/X10C. Après plusieurs années d'inactivité, la commission a été réactivée il y a deux ans, sous l'impulsion de quelques acteurs motivés. L'objectif de cette communication est de mettre en avant la plus-value de la normalisation dans l'activité d'hydrométrie, à travers quelques exemples concrets de normes.

L'organisation de la normalisation au plan national et international peut sembler complexe. Une présentation des différentes structures de normalisation est donc proposée, ainsi que le processus d'élaboration d'une norme.

Mots-clés : Normalisation, hydrométrie, AFNOR, Commission X10C, ISO, CEN

\section{Hydrometry and standardization}

\begin{abstract}
Normalization allows to define a common language among the actors in a particular field, to clarify, to harmonize practices and to define the level of quality, safety and less environmental impact of practices.

In France, in the field of hydrometry, the normalization work is carried out by the AFNOR / X10C commission. After several years of inactivity, the commission was recently reactivated two years ago, under the impulse of a few motivated actors. The objective of this paper is to highlight the added value of standardization in the hydrometry activity, through a few concrete examples of standards.

The organization of standardization at national and international level may seem complex. A presentation of the different standardization structures is therefore proposed, as well as the process of developing a standard.
\end{abstract}

Key-words: standardization, hydrometry, AFNOR (French national organization for standardization), X10C committee, ISO, CEN

\section{INTRODUCTION}

La gestion quantitative des ressources en eaux de surface et souterraines et la protection des milieux aquatiques nécessitent de disposer de données hydrométriques de qualité sur l'ensemble du cycle de l'eau. Ces données permettent l'élaboration de règles de gestion de la ressource (Plans de gestion de crise - PPRI -, débits de référence, bulletins de situation, arrêtés), le dimensionnement et la gestion d'ouvrages, ainsi que l'alimentation de modèles hydrologiques (calage, prévisions). La condition préalable à ces applications est de disposer de longues séries chronologiques pour produire des statistiques vraisemblables. L'acquisition, la validation et la mise à disposition des données hydrométriques nécessitent une méthodologie fiable et reproductible, à même de fournir aussi des informations sur les incertitudes.

La normalisation permet de définir un langage commun entre les acteurs d'un domaine particulier, de clarifier, d'harmoniser les pratiques et de définir le niveau de qualité, de sécurité, de moindre impact environnemental des pratiques. Une norme traduit un engagement à satisfaire un niveau de qualité et de sécurité reconnu et approuvé. A ce jour, les hydromètres francophones se retrouvent principalement autour de deux documents ayant fait l'objet de consensus 
inter organismes : la charte qualité hydrométrique [SCHAPI, 2017] et le guide d'utilisation ADCP [Le Coz et al., 2008]. Ces deux documents ont permis de fédérer l'ensemble des hydromètres à des pratiques homogènes et reconnues de tous.

En France, les travaux sur la discipline «Hydrométrie» sont suivis par la commission AFNOR/X10C «Hydrométrie» qui a repris son activité depuis 2014 et regroupe une dizaine de parties prenantes. Un des objectifs de cette commission est de mieux faire connaître les normes et leur utilité auprès de la communauté des hydromètres francophones, et de promouvoir leur utilisation. Un autre objectif avec une plus-value à plus long terme est la reconnaissance du savoir-faire français en hydrométrie qui peut être utile notamment lors du règlement de contentieux faisant intervenir des experts internationaux sur de la critique de données.

L'objet de la communication est de présenter, dans un premier temps, à travers quatre exemples concrets, la plus-value apportée par la normalisation. Dans un deuxième temps, le fonctionnement et l'organisation de la normalisation en hydrométrie, aussi bien au plan national qu'international, sont détaillés à travers les aspects suivants : présentation du rôle de l'AFNOR, le processus d'élaboration d'une norme, les comités de normalisation, la diffusion et l'accès aux normes.

\section{EXEMPLES DE NORMES EMBLÉMATIQUES EN HYDROMÉTRIE}

\section{II.1. ISO 772 : vocabulaire et notations (parler la même langue)}

Comme l'écrivait Albert Camus, « Mal nommer un objet, c'est ajouter au malheur de ce monde », et l'hydrométrie en est un bon exemple, tant les termes utilisés en français, en anglais ou dans les autres langues pour désigner un même concept ou un même objet sont divers et entraînent couramment des confusions entre opérateurs, même s'ils partagent la même langue. La normalisation du vocabulaire et des notations en hydrométrie fait l'objet de la norme NF EN ISO 772 [ISO, 2011], qui présente un grand nombre d'entrées techniques, avec définitions en anglais et en français et des index alphabétiques. Les entrées sont classées en huit chapitres :

1. Termes généraux

2. Méthodes d'exploration du champ des vitesses

3. Structures de mesurage de l'écoulement

4. Méthodes par dilution

5. Instruments et équipements

6. Transport des sédiments

7. Incertitudes des calculs hydrométriques

8. Eau souterraine

Poser des définitions claires pour parler la même langue est évidemment essentiel pour éviter des confusions et faciliter les échanges entre organismes, au niveau national comme international. Il est notamment plus qu'utile de se coordonner entre pays francophones pour établir des définitions communes, tout particulièrement pour désigner les nouvelles technologies et méthodologies, qui arrivent le plus souvent avec un vocabulaire anglais, voire spécifiquement anglo-américain. Les pays anglophones présentent aussi une certaine hétérogénéité de vocabulaire, comme en témoigne le terme " gauging " (jaugeage) employé dans certains pays «britanniques » (Royaume-Uni, Australie, Nouvelle-Zélande) mais pas en Amérique du Nord, où l'expression « discharge measurement » est utilisée à la place.
La norme NF EN ISO 772 comporte naturellement plus de définitions sur les techniques traditionnelles que sur les technologies plus récentes. Il faut reconnaître que les Québécois, par leur situation linguistique et géographique, ont une grande expérience dans l'art de traduire de nouveaux termes et expressions anglaises, et souvent une longueur d'avance sur les francophones européens en la matière. Lors de la consultation de 2016 sur la révision de la norme NF EN ISO 772, la commission AFNOR X10C a proposé d'ajouter l'entrée suivante sur la « index velocity method » introduite par l'USGS [Levesque et Oberg, 2012]. Elle a naturellement repris la traduction de cette expression qui est en usage courant au Québec, traduction à la fois originale et précise, dans le respect du français « traditionnel »:

\section{Méthode de la vitesse témoin}

Méthode, employée pour une station hydrométrique à mesure de vitesse en continu, permettant de calculer le débit du cours d'eau comme le produit de la surface mouillée (calculée à partir d'une relation hauteur-surface) et de la vitesse moyenne sur la section (calculée à partir d'une relation entre la vitesse témoin mesurée et la vitesse moyenne sur la section).

\section{Index velocity method}

Method used at hydrometric stations with continuous velocity measurements to compute the stream discharge as the product of the wetted area, computed with a stage-area rating, and of the cross-sectional average velocity, computed from a rating between the measured index velocity and the cross-sectional average velocity.

D'autres débats linguistiques sont plus anecdotiques, mais typiques de la diversité des usages et du problème des marques commerciales qui passent dans le langage courant par antonomase. On se souvient par exemple de l'examen de la question cruciale de la traduction souhaitable de « waders » en français (issu de « to wade ", marcher dans l'eau, patauger, traverser à gué). En France, c'est le terme le plus couramment utilisé, souvent avec la prononciation française « ouadeurses » : simple, pratique, élégant, comme l'objet en question. En France et au Québec, on trouve de nombreuses autres expressions, allant de «pantalon de pêche " à " bottes de pêche ", " bottes-culotte ", " bottes-pantalon ", ou encore " pontonnière ", apparemment utilisé en spéléologie...

La définition même de l'Hydrométrie et de ceux qui la pratiquent ne fait pas consensus ! La plupart d'" entre nous " restreignent l'Hydrométrie à la mesure des niveaux, vitesses et débits des cours d'eau naturels ou artificiels, mais l'ISO et l'OMM y ajoutent la mesure des écoulements souterrains et des flux de sédiments, voire même l'ensemble des mesures liées au cycle de l'eau (dont l'humidité des sols, les précipitations, l'évapotranspiration...). Dans les dictionnaires, " hydromètre " désigne selon les domaines : un compteur d'eau, un appareil servant à mesurer la densité, la pesanteur, la pression des liquides, un insecte vivant à la surface des petits plans d'eau, une accumulation de liquide séreux, non purulent, dans l'utérus... On ne trouve pas l'opérateur d'hydrométrie, mais on pourrait l'ajouter à la galerie, car c'est actuellement le terme le plus accepté en France. On trouve aussi « hydrométricien » et même " hydrométriste » sous la plume d'auteurs très sérieux, autres néologismes non consacrés et absents des dictionnaires. Le premier semble se référer à l'Hydrométrique et non l'Hydrométrie (cf. obstétrique, mathématique, optique... qui donnent des noms en -icien). Le deuxième est franchement moche et rime avec triste... 
L'OMM a consacré le terme « field hydrologist » (hydrologue de terrain), précis et clair mais un peu long, tandis qu' " hydrologue » peut sembler pas assez spécialisé. Mais on peut aussi souvent employer "opérateur » ou « jaugeur » selon le contexte. Les Américains du Nord (USA, Canada) et les Australiens (mais pas les Néo-Zélandais!) ont tendance à appeler improprement les « hydrologues de terrain » des " hydrographers », sans doute parce qu'ils produisent des " hydrographs », ce qui en anglais désigne toute série temporelle (ou chronique) hydrologique, que ce soit de débit ( discharge hydrograph » = " hydrogramme » en français), de hauteur (« stage hydrograph »= « limnigramme » en français) ou de tout autre paramètre mesuré en rivière. Certains anglophones natifs combattent cet usage impropre mais on le retrouve malheureusement dans le nom de certaines associations professionnelles comme l'AHA (Australian Hydrographers Association) et le NASH (North-American Association of Stream Hydrographers). Dans le monde entier, " hydrographie " et « hydrographe " désignent les mesures bathymétriques, principalement en domaine maritime, éventuellement fluvial.

Lors de la consultation de 2016 sur la révision de la norme NF EN ISO 772, la commission AFNOR X10C a donc proposé de clarifier les choses en ajoutant l'entrée suivante :

Hydromètre / Hydrologue de terrain / Technicien d'hydrométrie Opérateur professionnel spécialisé dans la mesure et le calcul des débits des cours d'eau, en particulier les opérations de jaugeage, la gestion des stations hydrométriques et l'établissement des courbes de tarage et des limnigrammes et hydrogrammes.

\section{Field hydrologist / Hydrometry technologist}

Professional operator specialising in streamflow measurement and computation, in particular streamganging operations, gauging station management, and the establishment of rating curves and stage and discharge times series.

La commission a également suggéré de dédier une entrée au terme " courantomètre / current meter », très courant en hydrométrie mais absent du glossaire. Il pose en réalité problème, car beaucoup de professionnels en France réservent le terme de " courantomètre » (ou « couranto ») aux courantomètres électromagnétiques, alors que pour le reste du monde « current meter » est synonyme de " vélocimètre » et désigne tout instrument permettant de mesurer un courant, c'est-à-dire la vitesse d'un écoulement, qu'il soit mécanique (moulinet), électro-magnétique (induction), radar ou acoustique (à effet Doppler). Il est possible que quand les premiers courantomètres électro-magnétiques sont arrivés en France, le mot " courantomètre » soit apparu avec (les courantomètres mécaniques à hélice étant traditionnellement appelés « moulinets ») et les opérateurs ont pensé que cela était réservé à des appareils mesurant un courant électrique (ce qui n'est en fait pas précisément le cas des courantomètres électro-magnétiques, qui en raison de l'effet Hall mesurent un courant à partir d'une mesure de tension et non de courant électrique...). La confusion sémantique perdure malgré une tendance à généraliser l'acception de courantomètre.

\section{II.2. ISO 748 : exploration du champ des vitesses (défense et amélioration des protocoles de mesure)}

La norme NF EN ISO 748 [ISO, 2009] est souvent considérée comme la norme la plus importante en Hydrométrie. Elle spécifie les procédures à suivre pour quantifier un débit par jaugeage par exploration du champ des vitesses à l'aide d'un courantomètre ou de flotteurs. Elle propose également une méthode de calcul des incertitudes, par application du GUM [JCGM, 2008] sous une forme simplifiée. Tant au travers des protocoles de déploiement sur le terrain, de dépouillement que de calcul des incertitudes, cette norme est au centre des échanges sur la justification et l'amélioration du processus fondamental de mesure, le jaugeage (même si d'autres techniques sont couramment employées, comme le jaugeage par dilution, par volumétrie ou par bateau mobile ADCP, qui ne rentrent pas dans le cadre de la norme NF EN ISO 748). Dans le cadre des opérations de Police de l'Eau menées par les agents assermentés de l'Agence française pour la biodiversité [Le Coz et al., 2011], cette norme et celles régissant les autres techniques de jaugeage sont fondamentales pour éviter la contestation des procès-verbaux dressés en cas de constat d'infraction aux obligations de respecter les débits réglementaires à l'aval d'ouvrages exploitant l'eau des cours d'eau (application de l'article L. 214-18 du Code de l'environnement).

Cette norme décrit un ensemble de protocoles de mesure acceptables, notamment trois méthodes pour mesurer et intégrer les vitesses ponctuelles sur une verticale de mesure : la méthode de distribution des vitesses (ou mode multi-points, avec intégration numérique ou graphique), la méthode à nombre de points limité (formules donnant la moyenne pondérée à appliquer à $1,2,3,5$ ou 6 points placés à des proportions normalisées de la profondeur d'eau, ou encore à 1 point mesuré près de la surface), et la méthode d'intégration (la vitesse moyenne est intégrée directement par déplacement lent du courantomètre de la surface au fond et retour, sur deux cycles consécutifs idéalement). La méthode de distribution des vitesses est la plus employée traditionnellement en France mais pratiquement pas en Amérique du Nord et en Australasie, où la méthode à 1 ou 2 points est la plus courante. La méthode d'intégration est utilisée en France, sur perche motorisée ou depuis un camion jaugeur, mais ces dispositifs ne sont pas employés couramment dans la plupart des pays.

Si toutes ces méthodes sont acceptées dans la norme, elles ne sont pas équivalentes et se traduisent par des pratiques très différentes. Pour schématiser, un jaugeage nord-américain comportera un grand nombre de verticales (au moins 20 en général) à 1 ou 2 points seulement, tandis qu'un jaugeage français comporte généralement moins de verticales (souvent entre 10 et 15 ) et un plus grand nombre de points sur chaque verticales. Le protocole français est souvent adapté en fonction de la configuration du site et de l'écoulement, à partir de l'expertise de l'opérateur.

Par le jeu des commissions de normalisation, les échanges techniques autour de la norme NF EN ISO 748, dont la révision est prévue en 2017, sont toujours intenses, afin que les pratiques des différents pays soient bien considérées dans le document, et que les différentes stratégies de mesure et de dépouillement des jaugeages soient bien expliquées et mises en regard. C'est là que l'analyse des incertitudes, et leur méthode de calcul, prennent toute leur importance opérationnelle, au-delà du simple exercice académique. En effet, l'analyse des incertitudes permet d'évaluer et d'améliorer les stratégies de mesure. Or, la méthode actuellement proposée dans la norme est reconnue comme insatisfaisante par la plupart des acteurs du domaine, notamment parce qu'elle ne rend pas assez compte de la diversité des environnements de mesure et propose un calcul simplifié à partir de composantes d'incertitude dont les valeurs indicatives tabulées dans l'Annexe E ne sont pas suffisamment justifiées, tracées et adaptées aux nouveaux instruments de mesure. 
Plus précisément, le résultat d'incertitude obtenu est très largement dominé par un terme forfaitaire reflétant l'incertitude d'intégration latérale des vitesses et profondeurs, dont la valeur est uniquement fonction du nombre de verticales, sans distinguer leur placement et le faciès de la section à mesurer. On peut noter toutefois que la norme NF EN ISO 748 prescrit de choisir le nombre de verticales de telle sorte que le débit dans chaque élément de section soit, dans la mesure du possible, inférieur à $5 \%$ du total et ne dépasse en aucun cas $10 \%$, ce qui conduit de fait à un nombre important de verticales et à une incertitude faible. Or, la pratique réelle actuelle reposant sur la réalisation d'un nombre plus limité de verticales, il est nécessaire de faire évoluer la norme pour proposer un calcul d'incertitude plus pertinent. Il est aussi reproché que le mode de calcul proposé favorise les protocoles à grand nombre de verticales au détriment de ceux à grand nombre de points sur la verticale, sans considérer que les premiers semblent en général mieux adaptés pour jauger des sections naturelles larges, tandis que les autres sont préférables pour des sections étroites et canalisées (cf. [ $\mathrm{Le} \mathrm{Coz}$ et al., 2011]).

Plusieurs méthodes alternatives de calcul des incertitudes des jaugeages par exploration du champ des vitesses ont donc été proposées récemment par différents groupes de recherche, en particulier les méthodes IVE (USGS, [Kiang et al., 2009]), Q+ (Irstea, [Le Coz et al., 2014]) et Flaure (EDF-DTG, [Despax et al., 2016]). Ces nouvelles méthodes ont principalement cherché à améliorer le calcul des composantes d'incertitude liées à l'intégration latérale et verticale des vitesses et des profondeurs, en lien avec la distribution des verticales et des points de mesure dans la section. Despax (2016) a comparé les résultats obtenus pour un grand nombre de jaugeages, principalement produits par EDF-DTG selon un protocole « français » et par l'USGS selon un protocole « nord-américain ». Les différentes méthodes estiment des niveaux d'incertitude très contrastés et les deux jeux de jaugeages USGS et EDF-DTG présentent des distributions d'incertitude différentes selon les méthodes (Figure 1). Au moment où la norme NF EN ISO 748 va bientôt être révisée, ces travaux de recherche seront très utiles pour éclairer les débats au niveau de la commission nationale AFNOR X10C comme au niveau du TC 113 de l'ISO.

\section{II.3. ISO 24578 : jaugeages ADCP}

(maîtrise et harmonisation des nouvelles technologies)

Un jaugeage par profileur Doppler consiste à explorer le champ des vitesses et la bathymétrie le long d'une section en travers d'un cours d'eau à partir d'un capteur ADCP immergé en surface et positionné sur une embarcation réalisant des traversées d'une rive à l'autre. Une traversée rive droite vers rive gauche, ou inversement, s'appelle un transect. Chaque transect fournit une valeur de débit.

L'utilisation de capteurs ADCP pour jauger les rivières a débuté en France en 1994. Le déploiement de cet équipement ainsi que l'incertitude de mesure associée étaient directement importée des USA, pays fournisseurs de ces appareils. La documentation existante était pauvre concernant l'incertitude sur la valeur de débit obtenue. A titre d'exemple, il fallait réaliser 6 transects pour n'en retenir que 4 en excluant le plus faible et le plus fort. Le débit était ensuite considéré comme fiable si la dispersion des 4 transects restant était inférieure à $5 \%$. La valeur d'incertitude admise était 5\% sans véritable fondement hormis l'expérience américaine.

C'était un bon début, toutefois pas suffisant au regard de la métrologie et de l'ensemble des profils de rivières françaises.

Des comparaisons interlaboratoires ADCP de grandes ampleurs ont été menées en France en $2009 / 2010 / 2012 / 2014 / 2016$. Ces essais sur sites ont permis d'affiner le protocole de mesures et d'élaborer un modèle de détermination d'incertitude [Le Coz et al., 2016].

D'autres pays ont également pris en main l'ADCP et ont élaboré leur propre protocole de mesures. Globalement tous ces protocoles se valent à quelques différences près.

L'objectif normatif est d'aboutir à un consensus technique visant à mettre à plat les bases communes au plus grand nombre tout en mettant en valeur les particularités de chacun pour que ce texte normatif apporte un plus et ne soit pas vécue comme une contrainte.

Le document normatif ISO/TR 24578 [ISO, 2012] existant répond en grande partie à cette problématique. Toutefois, les utilisateurs français n'y ont pas contribué et ce document nécessite une amélioration pour prendre en compte les avancées de ces dernières années. De plus, ce document est

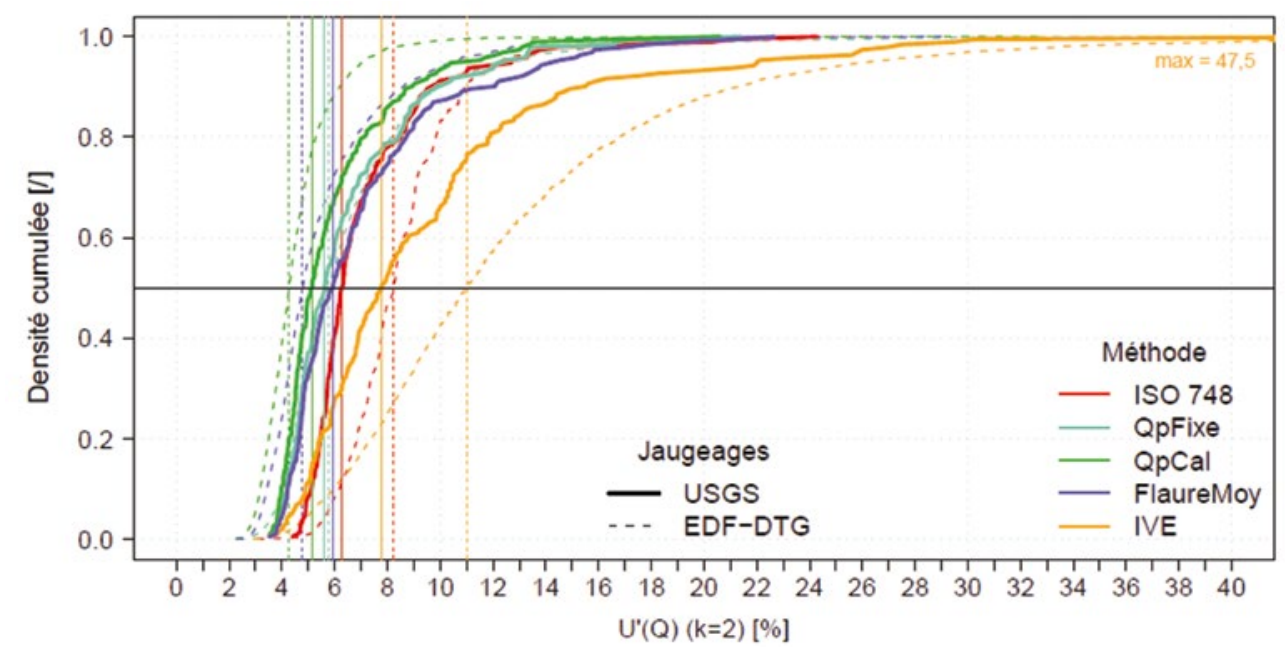

Figure 1 : Incertitudes élargies $U^{\prime}(Q)$ (au niveau de confiance de 95\%) selon quatre méthodes analytiques (ISO748, Q+ avec angle fixe ou calé, Flaure et IVE) appliquées à 192 jaugeages de l'USGS (Etats-Unis) et 3930 jaugeages d'EDF-DTG (France), présentées sous forme de distributions empiriques. Les lignes verticales colorées sont les valeurs médianes d'incertitude selon les différentes méthodes. La valeur maximale d'incertitude atteinte en appliquant la méthode IVE est de 47,5\% [Despax, 2016]. 
un rapport technique et n'a pas encore le statut de norme. L'objectif de l'ISO est de passer ce document au statut de norme. Ce document sera ensuite repris dans la collection française des normes, et traduit.

Le nouveau projet d'avril 2016 concernant la norme ISO/ TR 24578 devrait permettre de confronter l'ensemble des pratiques relatives au jaugeage ADCP de chacun (France et autres pays).

Ce document projet est déjà bien avancé, toutefois des points de différences méritent une attention particulière :

- Mode de déploiement : vitesse lente ou priorité sur la faible rotation du capteur quitte à se déplacer plus vite que l'écoulement?

- Durée de mesures : à adapter en fonction de la taille du cours d'eau et de la dynamique de l'écoulement ;

- Valeur aberrante : faut-il exclure systématiquement les données en dehors d'un seuil de dispersion ou seulement sur argumentaire physique d'écoulement ou de mesure ?

- Maintenance/contrôle préventif : contrôle tri-annuel via test en bassin de carène étalon COFRAC ou comparaison interlaboratoires ADCP ?

- Mesure invalide : sur quels critères exclure les transects avec ces mesures invalides (cellules ou verticales) ?

- Critère de validité d'un jaugeage : est-ce que la convergence des mesures est suffisante ou doit-on inclure d'autres critères, comme le ratio débit mesuré/débit total, le nombre de mesures invalides sur les cellules ou verticales, le fond mobile, etc. ?

Concernant les incertitudes de mesures du débit, plusieurs méthodes et logiciels associés ont été développées : Qrev par l'USGS [Mueller, 2016], OURSIN par CNR [Pierrefeu et al., 2017], QUANT par WSC [Moore et al, 2016] et RiverFlowUA par SFWMD [Gonzales et al., 2016]. Ces différents outils sont actuellement complémentaires. Ils mettent en évidence les points à « creuser » pour diminuer l'incertitude de mesure.

Les colloques internationaux et les comparaisons interlaboratoires ADCP montrent :

- l'importance d'un meilleur échange de pratiques pour aboutir à un texte consensuel normatif permettant d'avoir confiance dans la mesure de débit réalisée et ce quel que soit l'organisme ;

- de solides fondamentaux en métrologie pour l'expérience française via le Groupe Doppler Hydrométrique (GDH).

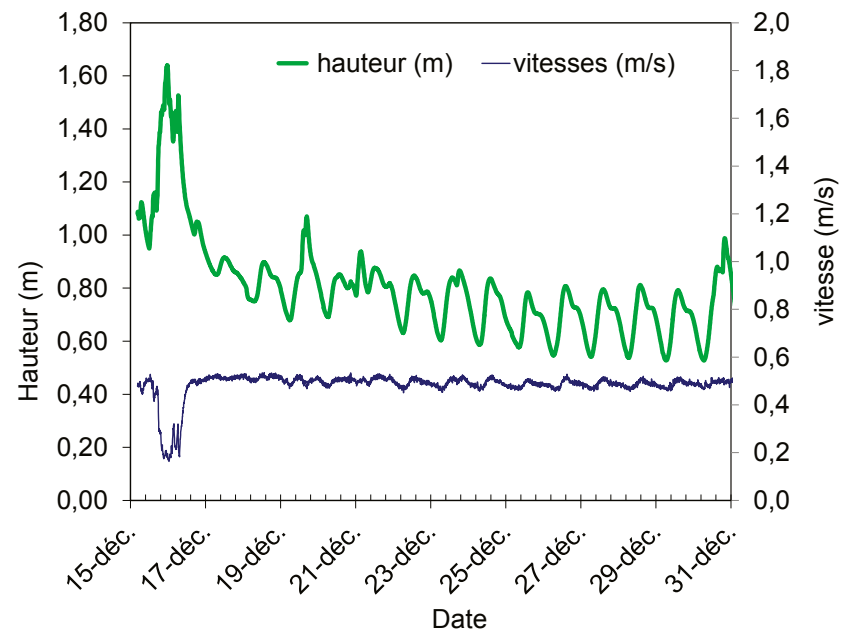

La révision de la norme ISO/TR 24578 est tout à fait dans le timing pour permettre aux utilisateurs français, via la commission AFNOR/X10C, de faire valoir ce qui leur parait nécessaire et essentiel à figer dans la norme. La liste des points ci-dessus est à prendre à titre d'exemples, d'autres points de convergence/différence seront débattus lors de la révision en cours qui est programmée sur une durée de 3 ans.

\section{II.4. ISO 15769 : Mesure en continu Doppler (station et contexte spécifique urbain)}

Les hauteurs d'eau en réseaux d'assainissement unitaires présentent souvent non seulement des cycles quotidiens de temps sec mais également des augmentations très importantes et rapides en temps de pluie. Si pour des raisons de simplicité d'installation et de fiabilité de la mesure, les installations ne mettant en œuvre que des mesures de hauteurs d'eau afin d'estimer les débits sont à privilégier, elles impliquent l'existence d'une relation permanente localement entre la hauteur d'eau et le débit. Dès que cette dernière n'est pas assurée, il devient nécessaire d'associer une mesure de vitesse longitudinale moyenne à la mesure de hauteur d'eau pour évaluer le débit liquide $\mathrm{Q}$ tel que avec : vitesse moyenne et : section de l'écoulement. La figure 2 montre le cas d'un site de réseau unitaire où la relation peut être considérée comme univoque si l'on se base sur une chronique d'une semaine de mesures (ici celle du 22 au 31 décembre) alors qu'une durée de mesures seulement doublée montre que la situation est plus complexe.

On comprend donc que pour cette station la détermination du débit implique de mesurer conjointement la hauteur et la vitesse de l'écoulement. Pour cela nous disposons de diverses technologies telles que, entre autres, les capteurs à effet Doppler, les profilomètres, les cordes de vitesse (temps de transit) [Larrarte et al., 2016].

Cependant, une particularité des réseaux d'assainissement urbain est que le débit connaît un cycle journalier de temps sec qui induit une grande variation de la hauteur dans le collecteur et qu'il s'avère donc délicat de choisir non seulement le matériel adapté à un site mais également comment l'installer sur un site donné. Si nous prenons le cas d'école représenté sur la figure 3 , on note que les capteurs rouges et noirs étant les mêmes, la position noire induit une sous évaluation

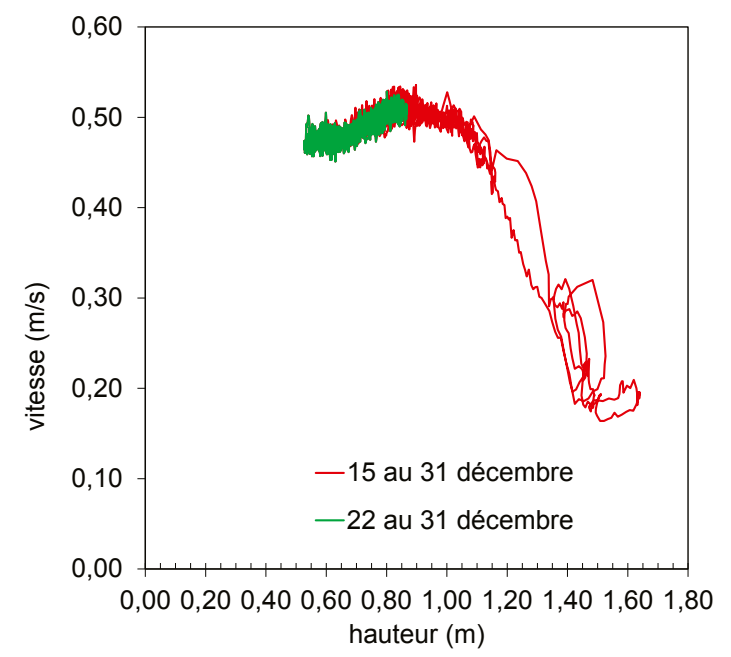

Figure 2 : Variation de la vitesse et de la hauteur d'eau en fonction du temps (gauche), variation de la vitesse moyenne en fonction de la hauteur d'eau selon deux périodes distinctes (droite). Station hydrométrique en réseau d'assainissement urbain unitaire. 


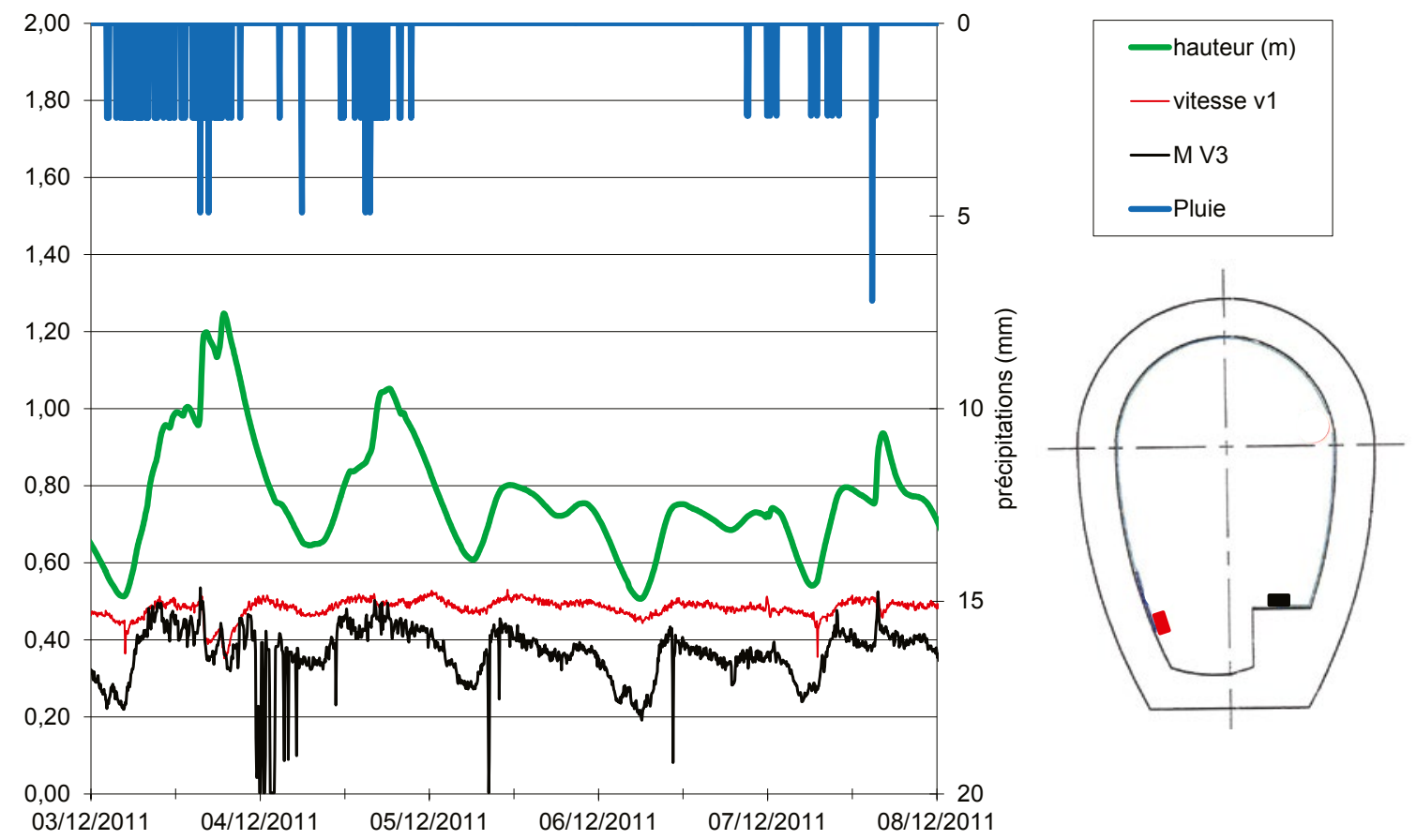

Figure 3 : Influence de la position des capteurs sur le suivi de la hauteur d'eau et de la vitesse dans un collecteur unitaire (à droite position des capteurs dans la section).

de la vitesse dès lors que la hauteur d'eau est inférieure à $0,5 \mathrm{~m}$. Cela s'explique par le fait que le faisceau ultrasonore intercepte une zone où la vitesse est moindre tant que la hauteur d'eau reste limitée (ici inférieure à $0,5 \mathrm{~m}$ ).

De la même manière, pour un site, une section transversale et une position donnée, le cône ultrasonore d'un capteur à effet Doppler ne mesure pas la vitesse dans la même partie d'une section mouillée [Larrarte et al., 2001] en fonction de ses caractéristiques, lesquelles varient substantiellement comme l'on montré Larrarte et al. [2008] dont les travaux prénormatifs avaient proposé un banc d'essai afin de déterminer ces principaux paramètres que sont l'angle d'émission, l'angle d'ouverture du faisceau, la portée, la pondération des échos, la fréquence d'émission (cf. figure 4).

La norme internationale ISO 15769 [ISO, 2010] reprend ces caractéristiques techniques des capteurs ultrasonores à effet Doppler et on ne peut qu'inciter les utilisateurs de ces matériels à s'informer des caractéristiques du matériel qu'ils souhaitent installer et de leurs adéquations avec le site concerné.

\section{FONCTIONNEMENT DE LA NORMALISATION EN HYDROMÉTRIE}

\section{III.1. L'AFNOR et les normes}

L'AFNOR est le représentant français de la normalisation au niveau du CEN et de l'ISO auprès desquels elle défend les positions françaises. Les normes peuvent donc être produites à différents niveaux : mondial (ISO), européen (CEN), ou national (NF). La figure 5 présente l'organisation de la normalisation du niveau national au niveau mondial.
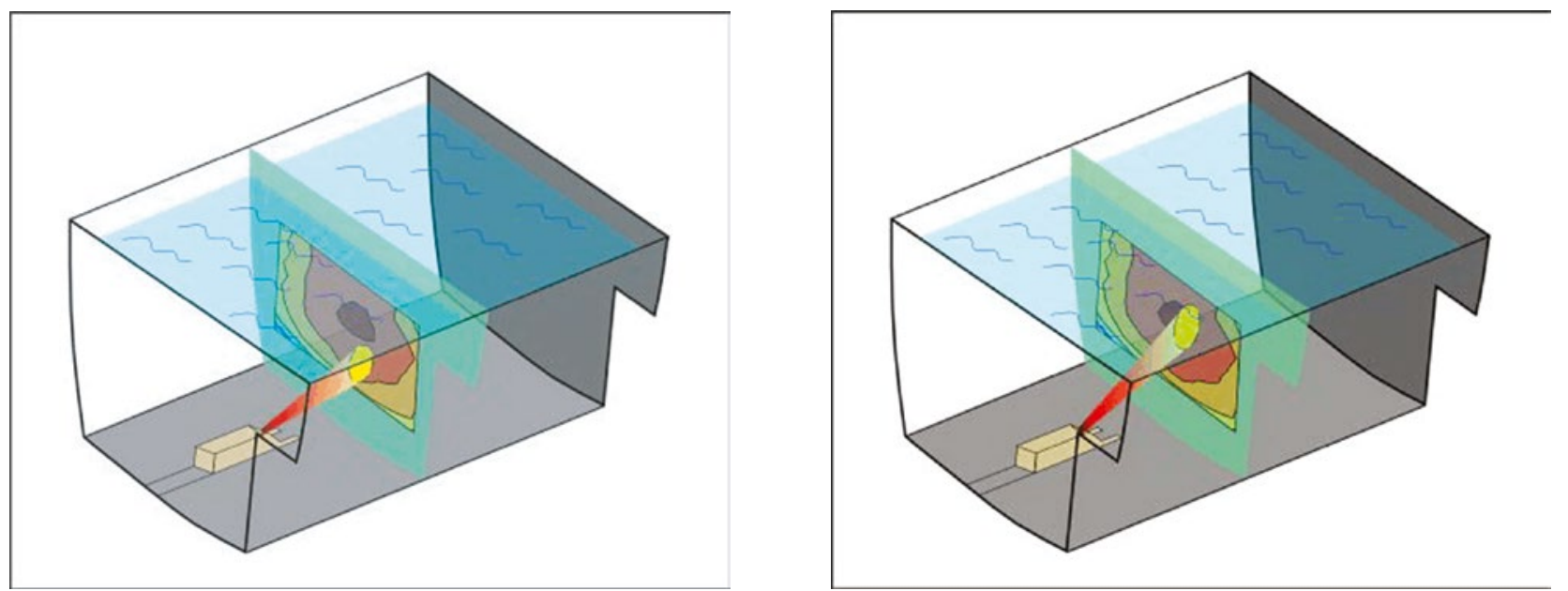

Figure 4 : Zone de mesure d'une vitesse moyenne en fonction des caractéristiques d'un capteur, ici influence de l'angle d'émission. 


\section{L'organisation de la normalisation}

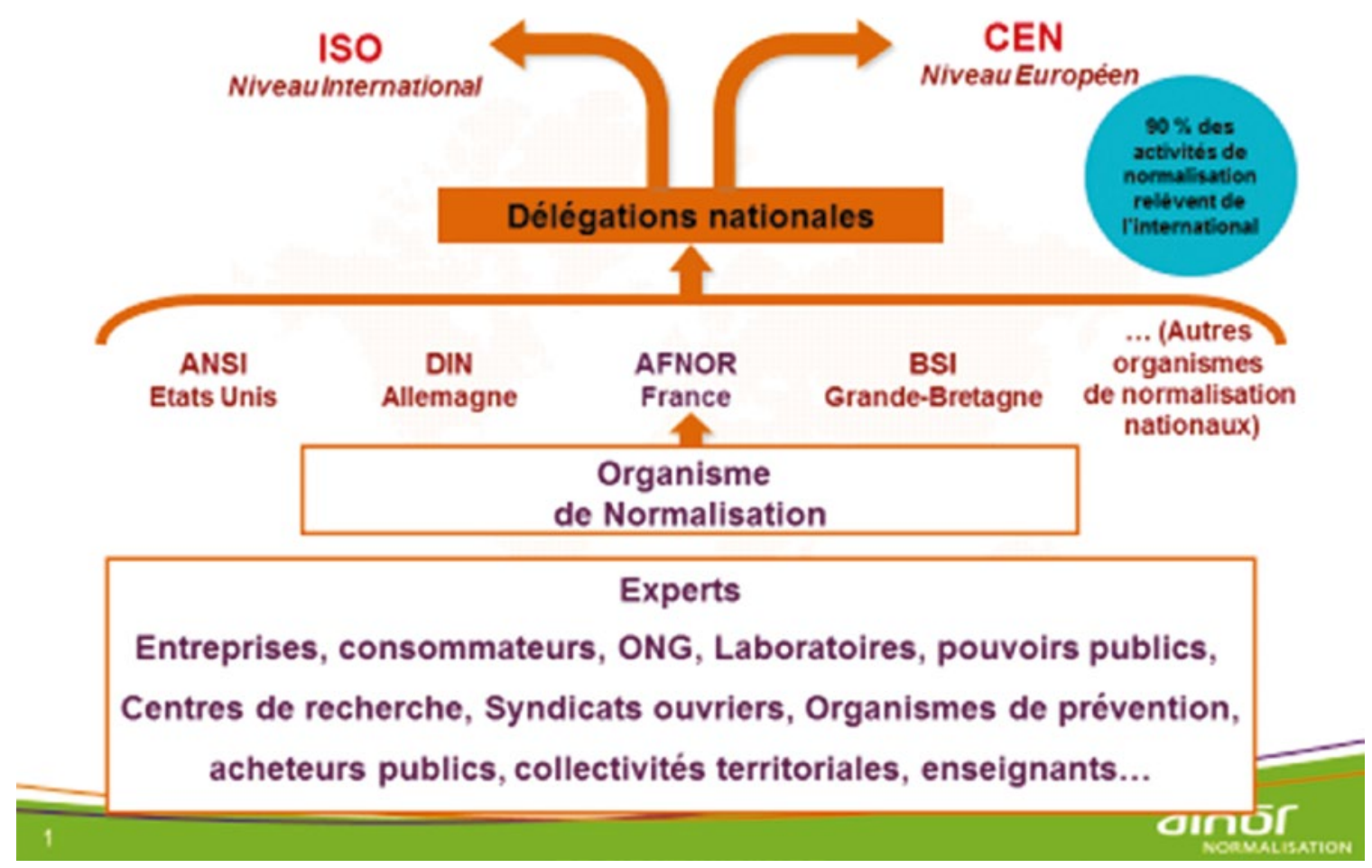

Figure 5 : Organisation de la normalisation (source AFNOR)

L'homologation en norme française est attestée, selon le niveau auquel elles ont été élaborées, par des préfixes tels que «NF ISO : normes internationales reprises en version française », « NF EN ISO : normes internationales reprises en normes européennes et en version française », « NF EN : normes européennes reprises en version française» ou « NF : textes nationaux français».

Les normes nationales doivent, soit reprendre les textes normatifs européens s'ils existent, soit être compatibles avec ces textes européens. Une norme nationale en contradiction ou portant sur le même sujet et le même contenu qu'une norme européenne est supprimée de la collection. Ces obligations ne valent pas pour les normes "seulement" internationales. $90 \%$ des normes françaises, tous domaines confondus, sont d'origine européenne ou internationale !

Il existe en France deux types de normes : les normes expérimentales et les normes homologuées.

- Les premières sont des référentiels «à l'essai » qu'il a paru nécessaire de soumettre à une période de mise à l'épreuve avant d'en conserver le contenu, tel que ou révisé. Elles sont reconnaissables à leur préfixe «XP». Leur équivalent à l'ISO ont pour préfixe "TS ». Ces textes devront soit devenir une norme soit être supprimés.

- Les secondes sont des référentiels dont la valeur technique est reconnue et officialisée par les pouvoirs publics, notamment pour servir de référence dans une réglementation, un marché public, une marque de certification, ... Élaborées et validées à l'issue d'une procédure largement ouverte à toutes les parties intéressées, elles sont l'expression d'un consensus. Les normes homologuées portent le préfixe du niveau d'élaboration (NF, EN, ISO), suivi de leur numéro et de l'année de leur homologation.
Les normes sont toujours susceptibles d'évoluer. Elles peuvent être amendées, révisées, voire abrogées et remplacées ou non.

En France, l'élaboration de normes est assurée par des commissions de normalisation qui rassemblent l'expertise technique et la connaissance des acteurs concernés. Pour l'hydrométrie la commission est référencée sous le terme de X10C « Hydrométrie ».

\section{III.2. Les étapes d'élaboration d'une norme internationale}

L'élaboration d'une norme respecte un certain nombre d'étapes qui sont détaillées dans la figure 6. Il est important de noter que toute norme devant être reprise dans la collection nationale fait l'objet d'une enquête publique, publiée sur le site internet de l'AFNOR. Ainsi, la dernière enquête publique traitée par la commission X10C a concerné la norme ISO 6416 [ISO, 2015] relative aux stations de mesure de débit par la méthode du temps de transit ultrasonique. Une information ciblée a été réalisée, notamment via la liste de diffusion électronique du Groupe Doppler Hydrométrie. Une dizaine de commentaires techniques ont été collectés, examinés par la commission X10C, et remontés au TC113 de l'ISO.

\section{III.3. Les comités de normalisation}

La commission de normalisation nationale X10C «Hydrométrie» de l'AFNOR intervient pour la normalisation « des méthodes, procédures, instruments et équipements se rapportant aux techniques pour la détermination hydrométrique du niveau, de la vitesse et des écoulements de l'eau, 


\section{Elaboration d'une norme internationale (ISO et IEC)}
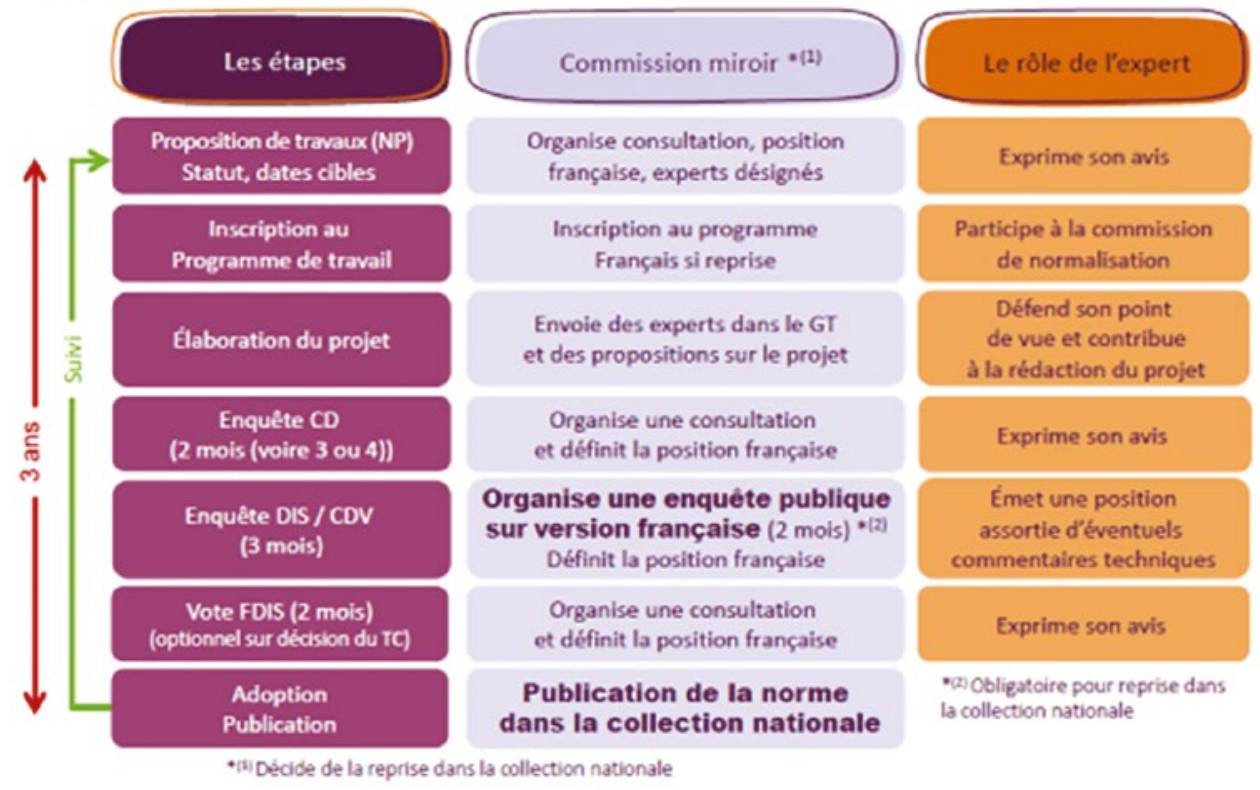

Figure 6 : Description des différentes étapes d'élaboration d'une norme internationale (source AFNOR). Les sigles sont explicités dans la partie $V$ "Nomenclature ».

des transports solides dans les canaux découverts, de la précipitation et de l'évapotranspiration, et de l'existence et du mouvement de la nappe superficielle, y compris :

- la terminologie et les symboles ;

- la recherche, l'évaluation, l'analyse, l'interprétation et la présentation des données ;

- l'évaluation des incertitudes. »

La commission donne son avis sur les normes ISO Hydrométrie lorsqu'elles sont en consultation. La reprise d'une norme internationale ISO dans la collection française fait l'objet d'un vote au sein de la commission X10C, alors que la reprise d'une norme européenne EN ou EN ISO dans la collection française est obligatoire.

Les membres de la commission X10C sont issus d'organismes publics (IFSTTAR, INERIS, IRD, IRSTEA, ONEMA, SCHAPI) ou privés (CACG, CNR, EDF DTG) impliqués dans le domaine de l'hydrométrie.

Pour l'ISO ou l'EN, l'équivalent des commissions portent le nom de comités techniques. En hydrométrie, il s'agit pour
l'ISO du TC113 et pour le CEN le TC318. La commission $\mathrm{X} 10 \mathrm{C}$ est donc chargée de suivre les travaux de ces comités, organisés en sous-comités ou groupes de travail, comme détaillé dans le tableau 1.

La commission $\mathrm{X} 10 \mathrm{C}$ a décidé de s'investir, dans un premier temps, sur les normes relatives aux $\mathrm{SC} 1, \mathrm{SC} 3$ et $\mathrm{SC} 5$ de l'ISO/TC 113.

Récemment (2013-2016), 16 normes ont fait l'objet d'un examen (révision, nouveau projet) et 2 ont fait l'objet d'une enquête publique.

\section{III.4. Diffusion et accès aux normes}

L'AFNOR propose plus de 30000 normes ou documents normatifs dans la collection française, accessibles par différents canaux et sous différents formats.

En effet on peut acheter les normes à l'unité, sous forme papiers, CD, pdf, ou html. Il existe aussi des solutions d'abonnement ou d'achat groupés.

Tableau 1 : Organisation des comités techniques internationaux et européens.

\begin{tabular}{|c|c|}
\hline $\begin{array}{l}\text { Comité technique international } \\
\text { ISO/TC } 113 \text { «Hydrométrie» et de ses sous-comités (SC) }\end{array}$ & $\begin{array}{l}\text { Comité technique européen } \\
\text { CEN TC } 318 \text { « Hydrométrie» et ses groupes de travail (WG) }\end{array}$ \\
\hline $\begin{array}{l}\text { SC } 1 \text { : Méthodes d'exploration du champ des vitesses } \\
\text { SC } 2 \text { : Structures mesurant le débit } \\
\text { SC } 3 \text { : Terminologie et symboles } \\
\text { SC } 5 \text { : Instruments, équipement et gestion des données } \\
\text { SC } 6 \text { : Transport solide } \\
\text { SC } 8 \text { : Nappe superficielle } \\
\text { Les travaux au sein du TC } 113 \text { sont sous la responsabilité de } \\
19 \text { pays participants (dont la France) }+16 \text { pays observateurs. }\end{array}$ & $\begin{array}{l}\text { WG } 5 \text { : Reference raingauge station } \\
\text { WG } 6 \text { : Guidelines for the selection of method } \\
\text { WG } 8 \text { : Groundwater } \\
\text { WG } 9 \text { : Water level measurement equipement } \\
\text { WG } 10 \text { : Uncertainties } \\
\text { Les travaux sont coordonnés par les représentants des } 28 \\
\text { pays membres de l'Union + Suisse, Norvège, Islande, } \\
\text { Turquie et Macédoine. }\end{array}$ \\
\hline
\end{tabular}


Les prix des normes varient de la dizaine à plusieurs centaines d'euros.

Les normes sont protégées par le droit d'auteur : lorsque vous achetez une norme, c'est pour un usage personnel ou documentaire. Vous ne pouvez en aucun cas la publier, la diffuser ou la vendre. La reproduction ou la diffusion même via les outils numériques sont strictement interdits.

Certaines normes sont disponibles en français alors que d'autres ne sont fournies qu'en langue anglaise.

Sur le site de la boutique en ligne de l'AFNOR (https://www.boutique.afnor.org/normes) sont disponibles plus de 80 normes liées à l'hydrométrie, dont une vingtaine françaises et plus de 30 internationales ISO. On trouve aussi une trentaine de normes anglaises (BSI) et une dizaine de normes allemandes (DIN), la plupart redondantes avec les normes ISO.

Trois solutions différentes permettent d'optimiser ou de gérer les coûts d'accès aux normes :

- Se constituer un recueil sur mesure avec les normes de votre choix (voir exemple https://www.boutique.afnor.org/ liste/recueils-de-normes-nouveautes/15/3/79/page/1/) qui permet de bénéficier d'une économie de $40 \%$ sur l'ensemble des normes du recueil. Cinq normes sont nécessaires, au minimum, pour constituer un recueil de normes et on ne peut dépasser 40 normes max (quel que soit le format choisi). Le format papier ne peut excéder 600 pages.

- Définir le périmètre de son abonnement via l'outil Saga Web de l'AFNOR (http://sagaweb.afnor.org/). Cet outil permet de constituer une base de données normative pour un travail collaboratif et un accès illimité aux normes de son choix en texte intégral. L'AFNOR propose une version d'essai sur son site. En exemple, l'Éducation Nationale a passé un accord avec l'AFNOR : dans un but strictement pédagogique, les établissements professionnels et technologiques de l'Éducation Nationale ont accès à l'ensemble des normes pour un coût inférieur à $3 €$ par élève et par an, avec un minimum de $350 €$ par établissement !

- Ouvrir un compte Normes et Produits d'Édition qui permet de régler ses achats sur la boutique en ligne AFNOR Éditions à l'aide d'un compte prépayé qui centralise l'ensemble des achats effectués. Un montant minimum de $1000 €$ est requis pour l'ouverture d'un Compte Normes et Produits d'Edition et le montant minimum de réapprovisionnement est de $500 €$.

On peut imaginer qu'en hydrométrie une politique d'accès spécifique aux normes puisse être mise en place (via le SCHAPI ?) via l'outil Saga Web ou bien en constituant un ou plusieurs recueils à définir entre hydromètres. L'OMM réfléchit également à la mise à disposition gratuite de certaines normes pour les services hydrologiques nationaux, en particulier dans les pays à faibles revenus.

Il apparaît en effet impossible d'envisager de promouvoir en interne (Groupe Doppler Hydrométrie) ne serait-ce qu'un résumé de bonne utilisation d'une norme donnée. Cette idée de s'approprier les normes via des documents plus simples et libres de droit se heurtent à la politique des droits d'auteur de l'AFNOR.

\section{CONCLUSIONS}

La normalisation en hydrométrie ou dans d'autres domaines de la mesure permet de se mettre d'accord collectivement sur des pratiques, des techniques et ainsi produire des résultats comparables partout dans le monde ou partout où les normes sont en vigueur. Ce cadre normatif, beaucoup plus prégnant dans l'industrie que dans nos domaines, s'impose maintenant de plus en plus dans l'hydrométrie. Les exemples choisis dans cet article reflètent bien la diversité des implications de la normalisation dans notre activité d'hydromètre.

La normalisation n'est pas le seul cadre structurant dans le domaine de l'hydrométrie. En France, on peut citer ainsi la Charte Qualité Hydrométrie [SCHAPI, 2017], dont l'objectif est d'inventorier et partager les bonnes pratiques dans tous les domaines de l'hydrométrie. Le Groupe Doppler Hydrométrie contribue également, en formalisant les échanges entre hydromètres et en capitalisant le retour d'expérience, à diffuser les bonnes pratiques. In fine, l'ensemble de ces démarches contribuent à améliorer la qualité des données produites.

Participer aux commissions, aux enquêtes publiques, suivre l'activité normative de notre domaine est un bon moyen de remettre en question nos pratiques, de confronter les approches locales avec celles de la communauté internationale et permettre ainsi de faire rayonner l'hydrométrie française comme un acteur du développement de l'hydrométrie.

Enfin, il est important de rappeler que la participation aux travaux de normalisation est ouverte à tout organisme ou personne désirant contribuer à l'amélioration des normes existantes ou à la proposition de nouvelles normes, à travers la participation aux enquêtes publiques, ou directement au sein de la commission AFNOR/X10C.

\section{NOMENCLATURE}

\section{ADCP : Acoustic Doppler Current Profiler}

AFNOR : Association Française de Normalisation http://norminfo.afnor.org : permet d'accéder aux normes et aux enquêtes publiques. Pour l'hydrométrie se rendre sur : Grand cycle de l'eau $>$ Hydrométrie

CACG : Compagnie d'aménagement des coteaux de Gascogne CD : Committee Draft (projet de comité)

CEN : Comité Européen de Normalisation http://www.cen. eu (pour l'hydrométrie se rendre sur standards.cen.eu $>$ technical bodies $>$ CEN/TC 318)

CNR : Compagnie nationale du Rhône

DIS : Draft for International Standard (projet de norme internationale)

DREAL : Direction régionale de l'environnement, de l'aménagement et du logement

EDF DTG : Electricité de France - Division technique générale

FDIS : Final Draft for International Standard (projet final de norme internationale)

IFSTTAR : Institut français des sciences et technologies des transports, de l'aménagement et des réseaux

INERIS : Institut national de l'environnement industriel et des risques

IRD : Institut de recherche pour le développement

IRSTEA : Institut national de recherche en sciences et technologies pour l'environnement et l'agriculture

ISO : International Organization for Standardization http:// www.iso.org (pour l'hydrométrie se rendre sur Elaboration des normes $>$ Comités techniques $>$ ISO/TC 113)

NP : New Project (nouveau projet)

OMM : Organisation météorologique mondiale

ONEMA : Office national de l'eau et des milieux aquatiques 
SCHAPI : Service central d'hydrométéorologie et d'appui à la prévision des inondations

SFWMD : South Florida Water Management District

TS : Technical Specification (spécification technique)

TR : Technical Report (rapport technique)

USGS : United States Geological Survey

WSC : Water Survey of Canada

\section{RÉFÉRENCES}

Despax, A. (2016) - Incertitude des mesures de débit des cours d'eau au courantomètre. Amélioration des méthodes analytiques et apports des essais interlaboratoires. Thèse de doctorat. Université Grenoble Alpes, 273 p.

Despax, A., Perret, C., Garçon, R., Hauet, A., Belleville, A., Le Coz, J. et Favre, A.-C. (2016) - Considering sampling strategy and cross-section complexity for estimating the uncertainty of discharge measurements using the velocity-area method. Journal of Hydrology, 533, 128-140.

Gonzales-Castro, J.A., Buzard, J. et Mohamed, A. (2016) RiverFlow UA - a package to estimate total uncertainty in ADCP discharge measurement by FOTSE - with an application in hydrometry,. River Flow 2016 - Constantinescu, Garcia \& Hanes, 715-723.

ISO (2009) - Norme NF EN ISO 748:2009. Hydrométrie Mesurage du débit des liquides dans les canaux découverts au moyen de débitmètres ou de flotteurs, $47 \mathrm{p}$.

ISO (2010) - Norme ISO 15769:2010. Hydrométrie - Lignes directrices pour l'application des compteurs acoustiques de vitesse utilisant l'effet Doppler et la corrélation d'échos, $61 \mathrm{p}$.

ISO (2012) - Norme ISO/TR 24578:2012. Hydrometry - Acoustic Doppler profiler-Method and application for measurement of flow in open channels, $45 \mathrm{p}$.

ISO (2011) - Norme NF EN ISO 772 :2011. Hydrométrie Vocabulaire et symboles, $153 \mathrm{p}$.

ISO (2015) - Norme ISO/DIS 6416:2015. Hydrométrie - Mesurage du débit par la méthode du temps de transit ultrasonique (temps de vol), $61 \mathrm{p}$.

JCGM (2008) - Evaluation of measurement data - Guide to the expression of uncertainty in measurement. Guide 100, BIPM, $132 \mathrm{p}$.

Kiang, J., Cohn, T. et Mason, R. (2009) - Quantifying uncertainty in discharge measurements. A new approach. In World
Environmental and Water Resources Congress 2009. American Society of Civil Engineers., 8 p.

Larrarte, F., Legendre, J.P., Sauvourel, Y., Gomin, F., Simon, R., Yviquel, G. ET Noel, C. (2001) - Vélocimétrie Doppler : mise au point d'un protocole d'essai en laboratoire et observations in situ. La Houille Blanche, 5, 67-74.

Larrarte, F., Bardiaux, J-B., Battaglia, P. et Joannis, C. (2008) - Acoustic Doppler flow-meters: a proposal to characterize their technical parameters. Flow Measurement and Instrumentation, 19, 261-267.

Larrarte, F., Joannis, C., Mignot, E., Rivière, N. et Lipeme KouYI, G. (2016) - Guide Technique - Fiabiliser les mesures de vitesse Représentativité spatiale des mesures en continu de vitesse et incertitudes sur les mesures de débit, . Guides techniques du projet MENTOR, $28 \mathrm{p}$.

Le Coz, J., Pierrefeu, G., Saysset, G., Brochot, J.-F. et Marchand, P. (2008) - Mesures hydrologiques par profileur Doppler. $164 \mathrm{p}$.

Le Coz, J., Camenen, B., Dramais, G., Ferry M., Rosique, J.-L. et Ribot-Bruno, J. (2011) - Contrôle des débits réglementaires, Application de l'article L. 214-18 du Code de l'environnement. Guide pratique Onema/Cemagref, 128 p.

Le Coz J., Bechon P.-M., Camenen B. et Dramais G. (2014) Quantification des incertitudes sur les jaugeages par exploration du champ des vitesses. La Houille Blanche, 5 , 31-39.

Le Coz, J., Blanquart, B., Pobanz, K., Dramais, G., Pierrefeu, G., Hauet, A. et Despax, A. (2016) - Estimating the uncertainty of stream gauging techniques using in situ collaborative interlaborary experiments. J.Hydraul.Eng, $15 \mathrm{p}$.

LeVesque, V. A. et OBerg, K. A. (2012) - Computing discharge using the index velocity method. U.S. Geol. Surv. Tech. Methods, 3-A23, 148 p.

Moore, S.A., Jamieson, E.C., Rainville, F., Rennie, C.D. et Mueller, D. (2016) - Monte Carlo approach for uncertainty analysis of Acoustic Doppler Current Profiler Discharge measurement by moving boat. J.Hydraul.Eng, $15 \mathrm{p}$.

Mueller, D.S. (2016) - Consistent and efficient processing of ADCP streamflow measurements. River Flow 2016 Constantinescu, Garcia \& Hanes, 655-663.

Pierrefeu, G., Bompart, G., Berthet, T. et Leboursicaud, R. (2017) - OURSIN : Outil de Répartition deS Incertitudes de mesure de débit par ADCP mobile. Congrès SHF " Hydrométrie 2017 », Lyon, 14-15 mars 2017.

SCHAPI (2017) - Charte Qualité Hydrométrie. 